\title{
Hybrid WDM/OCDMA system to support differentiated services in access network
}

\begin{abstract}
This paper presents a new scheme of WDM/OCDMA system that capable of providing differentiated services in access network. We implement the proposed double-weight (DW) code in this system. The double-weight (DW) code has a fixed weight of 2 and exists for every natural number, $n$. In spectral-amplitude-coding optical code division multiple access (CDMA) systems, DW code has an ideal cross correlation to eliminate multiuser interference and suppress the noise. We analyze the performance of the system by evaluating the bit error rate (BER) of different services.

Keyword: Bit error rate (BER); Double-Weight Code (DW); Optical code-division multiple access (OCDMA); Wavelength division multiple access (WDMA); Wavelength division multiplexing (WDM)
\end{abstract}

